\title{
EDUCAÇÃO EM SAÚDE NAS OFICINAS TERAPÊUTICAS DO CENTRO DE ATENÇÃO PSICOSSOCIAL: RELATO DE EXPERIÊNCIA NO ESTÁGIO SUPERVISIONADO EM SAÚDE MENTAL
}

\author{
Divanda Cruz ROCHA ${ }^{1}$ \\ Fabiana Paulino ALVES ${ }^{2}$ \\ Daiane de QUEIROZ ${ }^{3}$ \\ Elisângela Braga de AZEVEDO ${ }^{4}$ \\ Alessandra Carvalho LIMA ${ }^{5}$ \\ ${ }^{1}$ Enfermeira. Mestranda em recursos Naturais pela Universidade Federal de Campina Grande - PB (UFCG). Professora
do Departamento de Enfermagem da Universidade Estadual da Paraíba (UEPB). E-mail: divandac@ hotmail.com.
${ }^{2}$ Enfermeira. Mestre em Saúde Pública pela UEPB.
${ }^{3}$ Enfermeira. Mestre em Saúde Coletiva pela UEPB.
${ }^{4}$ Enfermeira. Doutoranda em Enfermagem pelo Programa de Pós-Graduação da Universidade Federal da Paraíba
(UFPB).
${ }^{5}$ Graduanda pelo Departamento de Enfermagem da Faculdade de Ciências Médicas de Campina Grande-PB (FCM).
}

Recebido em: 30/05/2014 - Aprovado em: 18/09/2014 - Disponibilizado em: 15/12/2014

\begin{abstract}
RESUMO:
A importância do estágio prático não se resume à integração do aluno ao mercado de trabalho ou ao aprimoramento de suas habilidades no âmbito profissional, trata-se também de um aspecto relevante na formação da pessoa. Objetivou-se neste relato descrever a experiência por uma docente e discentes da graduação do Curso de enfermagem no estágio supervisionado IV em saúde mental da Universidade Estadual da Paraíba, nas atividades de educação em saúde nas oficinas terapêuticas do Centro de Atenção Psicossocial II, no município de Campina Grande-PB. Trata-se de um estudo descritivo do tipo relato de experiência. A população alvo foram os usuários atendidos neste serviço, no turno da manhã. Foi realizado no período de fevereiro a junho de 2012, com um total de 24 discentes. O estágio Supervisionado em Saúde Mental possibilitou aos discentes uma oportunidade de desenvolverem atividades direcionadas para assistência dos usuários com sofrimento psíquico, buscando qualificação e humanização em seu atendimento, sob um novo enfoque de sistematização da assistência de enfermagem na prática, e com uma postura ética direcionada a estes clientes. Na avaliação final de cada subgrupo de estágio, eles expressaram que a Saúde mental deve ser trabalhada de forma dinâmica, com estratégias inovadoras e motivadoras, e que todos devem fazer parte da construção deste novo conhecimento. É importante destacar, que no "ensino/ aprendizagem devem-se criar espaços para os processos reflexivos e para a construção da autonomia responsável pelo discente".
\end{abstract}

PALAVRAS-CHAVES: Saúde mental, Educação em saúde, Assistência de enfermagem. Metodologia de ensino. Estágio supervisionado.

\section{HEALTH EDUCATION WORKSHOPS IN THERAPEUTIC CENTER OF PSYCHOSOCIAL CARE:} EXPERIENCE REPORT ON MENTAL HEALTH IN SUPERVISED

\section{ABSTRACT:}

The importance of practical training is not limited to the student's integration into the labor market or to improve their skills in a professional context, it is also a relevant aspect in the formation of the person. The objective of this report 
describe the experience of a teaching and student graduation course in nursing supervised stage IV in Mental Health at the State University of Paraiba, in the health education activities in therapeutic workshops at the Center for Psychosocial Care II, the municipality Campina Grande- PB. This is a descriptive study of the experience report type. The target population were patients seen in this service, the morning shift. Was conducted from February to June 2012, a total of 24 students. The Supervised internship in Mental Health allowed the students an opportunity to develop activities aimed to assist users with psychological distress, seeking qualification and humanization in your care, under a new approach to systematization of nursing practice, and an ethical stance directed these customers. In the final evaluation of each subset of training, they expressed that mental health should be imaged dynamically with innovative and motivating strategies, and that everyone should be a part of building this new knowledge. It is important to note that the "teaching / learning spaces should be created for the reflexive processes and the construction of responsible autonomy by the student."

KEYWORDS: Mental Health, Health Education, Nursing care . Teaching methodology. Supervised

\section{INTRODUÇÃO}

De acordo com as Diretrizes Curriculares do Curso de Graduação em Enfermagem, na formação do Enfermeiro, além dos conteúdos teóricos e práticos desenvolvidos ao longo de sua formação, ficam os cursos obrigados a incluir no currículo o estágio supervisionado em hospitais gerais e especializados, ambulatórios, rede básica, serviços de saúde e comunidades. Um dos objetivos da disciplina Saúde Mental, é compreender a saúde mental numa perspectiva preventiva e de saúde pública. $\mathrm{O}$ processo educativo tem a finalidade de transmitir informações para a população, objetivando a conscientização a respeito dos agravos à saúde. No entanto, isto só irá acontecer se a pessoa que receber a informação ver isto como importante para si (BOUSSO, et al., 2000).

Em decorrência do cenário do estágio, faremos um breve relato sobre a Reforma Psiquiátrica. A Reforma Psiquiátrica brasileira, através da inserção das ações de saúde mental na saúde pública, possibilita novas abordagens, novos princípios, valores e olhares às pessoas em situação de sofrimento psíquico, impulsionando formas mais adequadas de cuidado a portadores de transtornos mentais no seu âmbito familiar, social e cultural (HIRDES, 2009).

A mesma autora relata que este modelo conta com uma rede de serviços e equipamentos variados tais como os Centros de Atenção Psicossocial (CAPS), que vem assumindo um papel estratégico na organização da rede comunitária de cuidados, na qual farão o direcionamento local das políticas e programas de Saúde Mental desenvolvendo projetos terapêuticos e comunitários, dispensando medicamentos e acompanhando usuários que moram em residências terapêuticas, assessorando e sendo retaguarda para os Agentes Comunitários de Saúde (ACS) e para a Estratégia Saúde da Família (ESF) no cuidado familiar. Segundo o Ministério da Saúde, a Política Nacional de Saúde Mental, apoiada na Lei 10.216/02 que dispõe sobre a proteção 
das pessoas portadoras de transtornos mentais, busca consolidar um modelo de atenção à saúde mental aberto e de base comunitária, isto é, que garante a livre circulação das pessoas com transtornos mentais pelos serviços, comunidade e cidade, oferecendo cuidados com base nos recursos que a comunidade dispõe (BRASIL, 2001).

Os direitos das pessoas portadoras de transtornos mentais estabelecidos por essa Lei compreendem: ter acesso ao melhor tratamento, ser tratada com humanidade e respeito, ser protegida de abusos e exploração, ter garantia de sigilo, receber informações, ter acesso aos meios de comunicação, ser tratada pelos meios menos invasivos possíveis (GUIMARÃES et al., 2010)

Para Brasil (2004) os CAPS se
diferenciam pelo porte, capacidade de
atendimento, clientela atendida e organizam-
se no país de acordo com o perfil
populacional dos municípios brasileiros.
Assim, estes serviços diferenciam-se como
CAPS I, CAPS II, CAPS III, CAPSi e CAPSad. Nessa perspectiva, o CAPS II (cenário da nossa experiência), é um serviço aberto para atendimento diário de adultos com transtornos mentais severos e persistentes: trata-se de equipamento importante para municípios com população com mais de 70 mil habitantes. São instituições destinadas a acolher os usuários com transtornos mentais, estimular sua integração social e familiar, apoiá-los em suas iniciativas de busca da autonomia, e oferecer-lhes atendimento multiprofissional, cujo objetivo é oferecer atendimento à população de sua área de abrangência, realizando o acompanhamento clínico e a reinserção social dos usuários pelo acesso ao trabalho, lazer, exercício dos direitos civis e fortalecimento dos laços familiares e comunitários. É um serviço de atendimento de saúde mental criado para ser substitutivo às internações em hospitais psiquiátricos.

O trabalho do Enfermeiro nos CAPS deve ser desenvolvido com os seguintes objetivos: planejar, programar, e avaliar a assistência de enfermagem, a cada usuário ou grupo de usuário; criar e manter o ambiente terapêutico voltado para a realização das diversas atividades do CAPS; atuar junto ao cliente, a família e a equipe no atendimento de suas necessidades básicas para obtenção de uma saúde, física e mental; e colaborar na formação e aperfeiçoamento de novos profissionais na área de saúde mental e demais profissionais interessados na área (BRASIL, 2004).

O Presente estudo relata atividades desenvolvidas pela docente responsável pelo Estágio Supervisionado IV em Saúde Mental, e discentes da graduação do Curso de Enfermagem da UEPB, em um dos CAPES, no município de Campina Grande - PB. Para BOUSSO, (2000) a importância do estágio 
prático não se resume à integração do aluno ao mercado de trabalho ou ao aprimoramento de suas habilidades no âmbito profissional, trata-se também de um aspecto relevante na formação da pessoa.

Desse modo, objetivou-se descrever a experiência entre uma docente, e discentes da graduação em enfermagem da Universidade Estadual da Paraíba no estágio supervisionado IV em saúde mental, nas atividades de educação em saúde nas oficinas terapêuticas do CAPS II. Buscando sensibilizá-los acerca do novo modelo de assistência dispensado em saúde mental.

\section{METODOLOGIA}

Trata-se de um estudo descritivo do tipo relato de experiência, ocorrido no município de Campina Grande-Paraíba, referente à prática de ensino sob forma de estágio supervisionado IV em Saúde Mental, desenvolvido nas oficinas terapêuticas do CAPS II. A população alvo foram os usuários atendidos pelo CAPS II no turno da manhã. O estágio foi realizado no período de fevereiro a junho de 2012, com um total de 24 discentes, subdivididos em três discentes por subgrupos. O período do estágio supervisionado foi de nove dias para cada subgrupo. Utilizaram-se estratégias como: abordagem participativa e problematizadora para o processo de ensinoaprendizagem, dinâmicas de grupo, atividades lúdicas, músicas. Os métodos participativos levam o estudante a vivenciar situações propícias que possibilitam sua conversão em um ente ativo, criador, capaz de contribuir com o desenvolvimento do entorno social e sua própria autotransformação (SANLER,2001,p.25). Dentro desta mesma visão, Corrêa et al., (2011) destaca em seu estudo que é fundamental criar espaços para os processos reflexivos e para a construção da autonomia responsável pelo estudante. A metodologia baseada em problemas tem sido utilizada em diferentes áreas do conhecimento, na busca de promover maior aquisição de conhecimento, e maior desenvolvimento de habilidades nos discentes (CYRINO e TORALLES-PEREIRA, 2004).

\section{RELATO DE EXPERIÊNCIA}

Para Morais (2009) um método de ensino, pode ser compreendido com um procedimento didático composto por fases e operações para se alcançar um objetivo.

Dentro deste cenário, é importante ressaltar que o estágio supervisionado em Saúde Mental tem por objetivo sensibilizar e capacitar o discente a prestar assistência de enfermagem às pessoas em sofrimento psíquico no nível de assistência primário, secundário e terciário. 
Dinâmica do estágio: No $1^{\circ}$ dia era feito um breve resgate sobre a Reforma Psiquiátrica, e um acolhimento de boas vindas para deixá-los mais tranquilos, pois, alguns alunos relataram "que tiveram até pesadelos na noite que antecedia o estágio". Foram informados como seriam as atividades desenvolvidas no estágio; cada subgrupo ficaria responsável em preparar duas atividades educativas com temas préestabelecidos ou solicitados pela equipe ou usuários, e cada discente faria uma consulta de enfermagem com um usuário, e implementaria o Processo de Enfermagem para este usuário. Após estes esclarecimentos, eram apresentados a equipe e usuários, e em seguida era feito um breve reconhecimento do campo de estágio.

Estratégia Pedagógica: Cada grupo era estimulado para usar sua criatividade na elaboração da atividade, para deixá-la dinâmica e atrativa, pois, nas atividades educativas utilizávamos a metodologia participativa e problematizadora, ou seja, os usuários faziam parte da construção do conhecimento, tinham liberdade para fazerem perguntas ou acrescentarem informações pertinentes ao tema abordado. Sempre iniciávamos as oficinas com uma dinâmica de grupo para apresentação. Logo em seguida fazíamos uma avaliação diagnóstica no grupo sobre o tema, a partir deste momento as atividades eram iniciadas. É importante destacar, que a linguagem utilizada era simples, porém, com fundamentos científicos. De acordo com Santos e Varela (2007) a avaliação representa um dos pontos vitais para o alcance de uma prática pedagógica competente. Dentro desta mesma visão, Luckesi (2002) apud Santos e Varela (2007) afirma que a avaliação é um recurso pedagógico útil e necessário para auxiliar cada educador e cada educando na busca e na construção de si mesmos e dos seus melhores modos de ser na vida.

Para Colombo e Berbel (2007) a Metodologia da Problematização dá sua contribuição à educação, ao possibilitar a aplicação à realidade, pois desencadeia uma transformação do real, acentuando o caráter pedagógico na construção de profissionais críticos e participantes.

Técnicas desenvolvidas para exposição dos temas: $1^{a}$ - Uma caixa de papelão contendo perguntas sobre o tema abordado, $2^{a}$ - Música para marcar o compasso das atividades da seguinte forma: quem estivesse com a caixa contendo as perguntas pertinentes ao tema no momento em que a música parava retirava uma pergunta, e fazia a leitura em voz alta e respondia, se não soubesse ler ou responder tinha ajuda dos outros usuários ou dos discentes. $\quad 3^{a}$ - Utilizávamos também, cartolinas, e revistas para construção de cartazes com colagens de figuras e textos 
pequenos pertinentes aos temas trabalhados nas oficinas terapêuticas. Em seguida, fazíamos uma roda de discussão onde todos os usuários eram estimulados a participarem ativamente da atividade, expressando seu conhecimento sobre a temática trabalhada através de figuras ou de sua fala. Todo material construído ficou na instituição para fixação dos temas abordados e uso posterior.

Músicas selecionadas para as atividades: $\mathrm{O}$ Que É, O Que É? (Gonzaguinha), Homenzinho Torto (Aline Barros), Tocando Em Frente ( P. Fernandes), É o Amor (Zezé e Luciano Camargo), Banho de Chuveiro ( T. Samba),Meteoro ( L. Santana),O Melhor de Deus Está Por Vir ( K. Lucas), Bate o Pé (Rionegro e Solimões),Sonhos ( C. Durán), É Proibido Fumar ( Roberto e Erasmo), Tindolelê (Xuxa), Epitáfio ( Titãs), e Aquarela (Toquinho).

Oliveira (2013) destaca em seu estudo que é consenso entre os pesquisadores da Neurociência do poder estimulador que a música exerce sobre as atividades cerebrais em consonância com o corpo, a alma e o espírito. Evidenciou-se durante as oficinas, que a participação dos usuários era mais espontânea quando trabalhávamos com música.

Temas abordados pelos discentes nas oficinas terapêuticas: Dengue, higiene e saúde, calendário vacinal do adulto, hipertensão, riscos do fumo e outras drogas, prevenção do câncer de próstata, de mama, e colo do útero, violência contra a mulher, diabetes, higiene bucal, alimentação saudável, ricos do sedentarismo, o valor de um sorriso e um abraço, vacina contra influenza e H1N1,sustentabilidade,prevenção de quedas em idosos, importância do lazer para qualidade de vida e importância dos psicofárrmacos para a manutenção da saúde de quem é portador de transtorno mental.

Os temas abordados foram escolhidos de acordo com as prioridades do grupo que foi trabalhado e de acordo com a agenda nacional do Ministério da Saúde. É relevante informar que o Ministério da Saúde elabora todos os anos um calendário nacional de saúde, para ser trabalhado com atividades educativas na Atenção Básica de Saúde com datas preestabelecidas, por exemplo: o dia do diabético, o dia do hipertenso, o dia do idoso, entre outros ( BRASIL, 2011).

\section{Recursos didáticos utilizados:}

Notebook, lápis de cor e hidrocor, televisão, DVD, máquina fotográfica ,caixa de som, microfone, instrumentos musicais (Sanfona, zabumba, triangulo e violão), papel ofício, pincel atômico preto, vermelho e azul, folhas de papel madeira, cartolinas, bombons, pirulito, brindes, revistas, jornal, tesouras, toca CDs e CDs, quadro de giz, caixa de papelão pequena e média, fita adesiva, frutas (de plástico e natural), cola, cartazes, folhetos e quadros do Ministério da saúde, boneco 
educativo ( para ensinar a escovação correta), tintas para pinturas, chuveiro (simulação de um banho), confetes ( para simbolizar a água),e sabonetes.

\section{CONCLUSÃO}

Pretendemos com este relato, estimular uma reflexão por parte dos docentes e discentes, acerca do novo modelo de assistência dispensada ao portador de sofrimento psíquico, cujo principio primordial é resguardar uma assistência humanizada, planejada e com profissionais qualificados.

O estágio Supervisionado IV em Saúde Mental possibilitou aos discentes uma oportunidade de desenvolverem atividades direcionadas para assistência dos usuários com sofrimento psíquico. A enfermagem vem reconquistando seu espaço dentro da nova assistência dispensada ao cliente com sofrimento psíquico, buscando qualificação e humanização em seu atendimento, sob um novo enfoque de sistematização da assistência de enfermagem na prática, e com uma postura ética direcionada a estes clientes.

É de suma importância sensibilizar os discentes, para desenvolverem um novo olhar para os portadores de sofrimento psíquicos, percebendo também, que o enfermeiro faz parte deste novo modelo de atenção voltado para a Saúde Mental.
Evitamos neste estágio, que o discente fosse apenas um mero observador, mas que ele fosse parte ativa na construção das atividades que seriam implementadas para está clientela durante o período de estágio nas oficinas terapêutica.

Percebemos com o decorrer do estágio, que a metodologia utilizada, possibilitou uma maior interação entre os discentes, usuários e equipe. Neste semestre percebemos que ocorreu também, um número reduzido de faltas por parte dos discentes. Há uma necessidade urgente de utilizarmos novas estratégias no estágio supervisionado em Saúde Mental, para que o mesmo se torne prazeroso, dinâmico e motivador.

$\mathrm{Na}$ avaliação final de cada subgrupo, “eles expressaram que a Saúde mental deve ser trabalhada de forma dinâmica, com estratégias inovadoras e motivadoras, que estimulem a assiduidade e participação dos mesmos, e que todos façam parte da construção e disseminação deste novo modelo de assistência, cujo objetivo é integrar este usuário ao meio social que convive". Concluímos este relato de experiência com a definição de Saúde Mental segundo a OMS "saúde mental é a capacidade de estabelecer relações harmoniosas com os demais e a contribuição construtiva nas modificações do ambiente físico e social". 


\section{REFERÊNCIAS}

BOUSSO, R.S. et al. Estágio curricular em

Enfermagem: transição de identidades.

Rev.Esc.Enf. 2000. USP, v. 34, n. 2, p.218.

Disponível em:

<http://www.scielo.br/pdf/reeusp/v34n2/v34n

2a13.pdf $>$ Acesso em 18 jul. 2012.

BRASIL. Ministério da Saúde. Secretaria de

Vigilância em Saúde. Plano de ações

estratégicas para o enfrentamento das

doenças crônicas não transmissíveis

(DCNT) no Brasil 2011-2022. Brasília. 2011.

BRASIL. Ministério da Saúde. Saúde Mental no SUS: os centros de atenção psicossocial.

2004. Disponível em:

<http://www.ccs.saude.gov.br/saude_mental/p df/sm_sus.pdf..$>$ Acesso em: 18 de jul. 2012.

BRASIL. Ministério da Saúde. Lei no 10.216, de 06 de abril de 2001. Dispõe sobre a proteção e os direitos das pessoas portadoras de transtornos mentais e redireciona o modelo assistencial em saúde mental. Antigo Projeto de lei Paulo Delgado. Diário Oficial da União, 09 de abr.2001.

CORRÊA, Adriana Katia et al . Metodologia problematizadora e suas implicações para a atuação docente: relato de experiência. Educ. rev., Belo Horizonte, v. 27, n. 3, Dec.

2011. Disponível em:

$<$ http://www.scielo.br/scielo.php?pid=S0102$\underline{46982011000300004 \& \text { script=sci_arttext }>}$

Acesso em 28 maio. 2014.

COLOMBO, A. A. BERBEL, N. A. N. A metodologia da problematização com o Arco de Maguerez e sua relação com os saberes de professores. Rev Semina: Ciências Sociais e Humanas, Londrina, v. 28, n. 2, p. 121-146, jul./dez. 2007. Disponível em: <http://www.uel.br/revistas/uel/index.php/se minasoc/article/view/3733> Acesso em 10 de maio.2014.

CYRINO, E.G.; TORALLES-PEREIRA, M. L. Trabalhando com estratégias de ensinoaprendizado por descoberta na área da saúde: a problematização e a aprendizagem baseada em problemas. Cad. Saúde Pública, Rio de Janeiro, v. 20, n. 3, June 2004. Disponível em: 〈http://www.scielo.br/pdf/csp/v20n3/15> Acesso em: 29 de maio. 2014.

GUIMARÂES, N.A. et al. O Tratamento ao Portador de Transtorno Mental: um diálogo com a Legislação Federal Brasileira (1935- 2001).Texto Contexto Enferm, Florianópolis, 2010 Abr-Jun; 19(2): 274-82, p. 281. Disponível em: <http://www.scielo.br/pdf/tce/v19n2/08.pdf $>$ Acesso em: 28 de maio. 2014. 
Enferm. 2004. USP, v. 34, n. 2, p. 191.

HIRDES, A. A Reforma Psiquiátrica no

Brasil: uma (re) visão. Revista Ciência \&

Saúde. v.14 n.1 Rio de Jan./

Fev. 2009,14(1):297-305,p.304.Disponível

em:

<http://www.scielo.br/scielo.php?pid=s1413-

$\underline{81232009000100036 \& \text { script }=\text { sci_arttext\&tlng }}$

=en > Acesso em 20 de jul.2012.

MORAIS, M. de F. A utilização de métodos participativos no ensino de Engenharia de Produção: o caso do curso de Engenharia de Produção Agroindustrial da FECILCAM. In:

IV Encontro de Produção Científica e

Tecnológica. Anais... Campo Mourão - PR. 2009. Disponível em:

$<\underline{\text { http://www.fecilcam.br/nupem/anais_iv_epc }}$ t/PDF/engenharias/04_MORAIS.pdf $>$ Acesso em 20 de maio 2014.

OLIVEIRA, P. A. de. Música e arteterapia como recurso terapêutico nas dificuldades de aprendizagem e desenvolvimento humano.

Constr. psicopedag., São Paulo, v. 21, n.

22, 2013 .Disponível em: <

http://pepsic.bvsalud.org/scielo.php?pid=S141 $\underline{5-69542013000100008 \& \text { script }=\text { sci_arttext }>}$

Acesso em: 10 de jan. 2014.

RUIZ, V.R.; LIMA, A.R.; MACHADO, A.L.

Educação em saúde para portadores de doença mental: relato de experiência. Rev. Esc.
Disponível em:

$<$ http://www.scielo.br/scielo.php?script=sci_a $\underline{\text { rttext\&pid }=S 0080-62342004000200010>}$ Acesso em18 jul. 2012.

\section{SANLER, Verônica Canfux. Métodos}

Participativos. Universidad de la Habana: CEPES, 2001.

SANTOS, M. R. dos. VARELA, S. A avaliação como um instrumento diagnóstico da construção do conhecimento nas séries iniciais do ensino fundamental. Revista Eletrônica de Educação. Ano I, No. 01, ago. / dez. 2007. Disponível em:

$<$ http://www.reveduc.ufscar.br/index.php/reve duc/search/titles> Acesso em:28 de maio.2014.

TOWNSEND, M. C. Enfermagem

Psiquiátrica: conceitos de cuidados. $3^{\text {nd. }}$ Rio de Janeiro. Guanabara Koogan. 2002. 